ARTICLE

https://doi.org/10.1038/s41467-019-12426-9

\title{
First-in-human trial of blood-brain barrier opening in amyotrophic lateral sclerosis using MR-guided focused ultrasound
}

Agessandro Abrahao (1) 1,2,3,11*, Ying Meng 2,3,4,11, Maheleth Llinas ${ }^{3,5}$, Yuexi Huang ${ }^{5}$, Clement Hamani ${ }^{2,3,4}$, Todd Mainprize $^{4}$, Isabelle Aubert (1) ${ }^{2,6}$, Chinthaka Heyn ${ }^{7,8}$, Sandra E. Black (1) 1,2,5,9, Kullervo Hynynen ${ }^{5,8,9,10}$, Nir Lipsman $2,3,4,12$ \& Lorne Zinman $1,2,12$

MR-guided focused ultrasound (MRgFUS) is an emerging technology that can accurately and transiently permeabilize the blood-brain barrier (BBB) for targeted drug delivery to the central nervous system. We conducted a single-arm, first-in-human trial to investigate the safety and feasibility of MRgFUS-induced BBB opening in eloquent primary motor cortex in four volunteers with amyotrophic lateral sclerosis (ALS). Here, we show successful BBB opening using MRgFUS as demonstrated by gadolinium leakage at the target site immediately after sonication in all subjects, which normalized 24 hours later. The procedure was well-tolerated with no serious clinical, radiologic or electroencephalographic adverse events. This study demonstrates that non-invasive BBB permeabilization over the motor cortex using MRgFUS is safe, feasible, and reversible in ALS subjects. In future, MRgFUS can be coupled with promising therapeutics providing a targeted delivery platform in ALS.

\footnotetext{
${ }^{1}$ Division of Neurology, Department of Medicine, Sunnybrook Health Sciences Centre, University of Toronto, Toronto, ON M4N 3M5, Canada. ${ }^{2}$ Hurvitz Brain Sciences Research Program, Sunnybrook Research Institute, Sunnybrook Health Sciences Centre, University of Toronto, Toronto, ON M4N 3M5, Canada. ${ }^{3}$ Harquail Centre for Neuromodulation, Sunnybrook Research Institute, Toronto, ON M4N 3M5, Canada. ${ }^{4}$ Division of Neurosurgery, Sunnybrook Health Sciences Centre, University of Toronto, Toronto, ON M4N 3M5, Canada. ${ }^{5}$ Sunnybrook Research Institute, Sunnybrook Health Sciences Centre, University of Toronto, Toronto, ON M4N 3M5, Canada. ${ }^{6}$ Department of Laboratory Medicine and Pathobiology, University of Toronto, Toronto, ON M5S 3H7, Canada. ${ }^{7}$ Department of Medical Imaging, Sunnybrook Health Sciences Centre, University of Toronto, Toronto, ON M4N 3M5, Canada. ${ }^{8}$ Odette Cancer Research, Sunnybrook Research Institute, Sunnybrook Health Sciences Centre, University of Toronto, Toronto, ON M4N 3M5, Canada. ${ }^{9}$ Institute of Biomaterials and Biomedical Engineering, University of Toronto, Toronto, ON M5S 3H7, Canada. ${ }^{10}$ Department of Medical Biophysics, University of Toronto, Toronto, ON M5S 3H7, Canada. ${ }^{11}$ These authors contributed equally: Agessandro Abrahao, Ying Meng. ${ }^{12}$ These authors jointly supervised this work: Nir Lipsman, Lorne Zinman. *email: agessandro.abrahao@sunnybrook.ca
} 
A myotrophic lateral sclerosis (ALS) remains a fatal neurodegenerative disease characterized by the progressive degeneration of both the upper and lower motor neurons (UMNs and LMNs). There is an incomplete understanding of the disease pathophysiology and current interventions only mildly slow disease progression. While preclinical studies in ALS rodent models have primarily focused on the distal-to-proximal motor neuron dying-back hypothesis ${ }^{1}$, recent data support a key pathological role of UMN in the motor cortex ${ }^{2}$. Signs of cortical neuronal and glial dysfunction have been observed early in the disease course and preceded spinal cord neuronal degeneration in ALS rodent models $s^{3,4}$, supporting a motor cortex driven dyingforward hypothesis ${ }^{2}$. Early cortical motor neuron dysfunction in ALS has also been demonstrated in human studies using transcranial magnetic stimulation, neuroimaging and electrophysiological measures ${ }^{2,5,6}$. Therefore, interventions that target cortical motor neurons alone or in combination with LMNdirected therapies may be essential in slowing or halting disease progression.

Access to the neurons and glia of the primary motor cortex remains challenging as the blood-brain barrier (BBB) limits the transfer of therapeutics from the intravascular compartment. While there is preclinical and post-mortem evidence of abnormal $\mathrm{BBB}$ ultrastructure in $\mathrm{ALS}^{7,8}$, the BBB still restricts the passage of drugs, antibodies, gene carriers and stem cells from the capillaries to the central nervous system $(\mathrm{CNS})^{9}$. Even riluzole, an approved drug for ALS, has limited availability in the brain tissue given BBB-related drug efflux mechanisms ${ }^{10}$.

Presently, the targeted delivery of biotherapeutics to the brain requires an invasive neurosurgical procedure, which can have serious complications. Promising biotherapeutic approaches to UMN pathology in ALS, including neural progenitor cells ${ }^{11}$ and adeno-associated virus (AAV) expressing small-hairpin RNA to suppress mutant superoxide dismutase-1 (SOD1) ${ }^{3}$, have been surgically injected into the motor cortex of ALS rodents, resulting in significantly increased lifespan. Stereotactic implantation of autologous $\mathrm{CD}_{133^{+}}$stem cells into the primary motor cortex has also been investigated in a small cohort of ALS subjects with limited success ${ }^{12}$. Alternatively, non-invasive delivery methods across the $\mathrm{BBB}$ via facilitated transcellular transport (e.g., drug modifications with transferrin or insulin receptors ${ }^{13}$ ), drug efflux inhibition (e.g., elacridar ${ }^{10}$ ), or diffuse BBB breakdown (e.g., mannitol) lead to widespread CNS uptake and potential off-target effects. Given the limitations of open surgery and non-targeted pharmacological delivery approaches, the development of a noninvasive, image-guided method to safely and temporarily open the $\mathrm{BBB}$ for targeted delivery would represent a paradigm shift in the treatment of ALS.

Transcranial magnetic resonance-guided focused ultrasound (MRgFUS) combined with intravenous ultrasound contrast (perflutren lipid microbubbles) has emerged as an incision-less technique to transiently open the BBB in targeted CNS regions ${ }^{14}$. The MRgFUS device is comprised of a multi-element phased array transducer system that can target virtually any brain region with millimetric accuracy, using real-time MRI feedback for tissue monitoring and intraoperative target guidance ${ }^{15}$. At higher frequencies, MRgFUS thalamotomy was found to be effective in treating medically refractory essential tremor in a randomized controlled trial ${ }^{16}$. At lower powers, ultrasound interacts with injected microbubbles resulting in transient mechanical disruption of the BBB without thermal injury ${ }^{17-19}$. This has been shown to enhance drug delivery to multiple brain regions in small-tolarge animals, including cortical targets ${ }^{20}$, the hippocampus ${ }^{21}$, striatum $^{21,22}$, and brainstem ${ }^{23}$. Although safe BBB opening in eloquent and non-eloquent brain regions has been demonstrated in subjects with high-grade gliomas using surgically implanted intracranial pulsed ultrasound device ${ }^{24,25}$, transcranial MRgFUS $\mathrm{BBB}$ opening is a non-invasive approach and has optimal spatial resolution for discrete and precise $\mathrm{BBB}$ targeting in eloquent regions, such as the primary motor cortex. In recent pilot clinical trials, non-invasive MRgFUS BBB opening in small and noneloquent regions in and around gliomas ${ }^{26}$ and in the frontal lobe of patients with Alzheimer's disease $(\mathrm{AD})^{14}$ was safe and transient.

This study represents the first-in-human trial testing the feasibility and safety of transient permeabilization of the BBB using MRgFUS in subjects with ALS. It is also the first attempt to apply this technology to eloquent cortical and subcortical tissue in human subjects as primary motor cortex was targeted using functional MRI (fMRI). Here, we show safe and reversible motor cortex BBB opening using MRgFUS at the target site immediately after sonication in all subjects without serious adverse events.

\section{Results}

Participants and target ascertainment. Four eligible subjects, two women and two men with median age 61 years (range: 56-70), diagnosed with ALS (probable El Escorial criteria) and clinical evidence of upper motor neuron dysfunction were enrolled in the study (Fig. 1). Table 1 lists their baseline demographics. Each subject's sonication targets (Supplementary Fig. 1) were individualized according to arm (Fig. 2a) or leg (Fig. 3a) fMRI activation in the primary motor cortex to ascertain the homunculus topography. Hand activation by subject two and three was spatially shifted laterally from what is typically described as the hand knob area of the precentral gyrus. Cortical atrophy of the hand functional region was also noted on visual inspection at baseline in subject three, who presented with armonset phenotype. Subject four, who had leg-onset ALS, revealed bilateral motor cortex activation on fMRI.

Outcomes. MRgFUS-mediated opening of the BBB was successful in all four subjects as judged by demonstration of gadolinium leakage directly at the sonication target (Figs. 2, 3) and further indicated by increases in normalized intensity ratio (IR) on MRI (Fig. 4). Notably, the IRs decreased one day following the procedure indicating reversible $\mathrm{BBB}$ permeability. The total target volume of $350 \mathrm{~mm}^{3}$ was permeabilized with 2-4 rounds of sonication with a median power of 8.0 (range: 6.0-10.0) W (Supplementary Table 1). Real-time MR thermometry monitoring did not detect temperature elevation.

$\mathrm{BBB}$ opening in the primary motor cortex was well tolerated with few mild-to-moderate procedure-related adverse events (AEs) (Table 2). There was no procedure- or device-related serious adverse events (SAEs) from intervention to 60 days of follow-up. Subjects tolerated the procedural time and intravenous microbubble injections, with no immediate tissue reaction such as hemorrhage or edema. Medications administered for patient's comfort and AE management during the intervention are listed in Supplementary Table 2. Median duration in the MRgFUS procedure was $66 \mathrm{~min}$ (range: 43-173), which included acquisition of planning sequences, targeting and sonications. Median duration of pre- and post-sonication MR scanning was $115 \mathrm{~min}$ (range: 90-147). No change in the neurological status was detected during the sonications in any of the patients and the 24$\mathrm{h}$ inpatient observation period was uneventful. All patients were discharged on the first post-operative day. In subject four, a transient and asymptomatic hyperintense sulcal and parenchymal fluid-attenuated inversion recovery (FLAIR) signal was observed in a portion of the sonicated region on the day 1 and resolving on day 7 MR scans, without any accompanying changes on T2, T2* gradient echo (GRE), or diffusion weight imaging (DWI) 


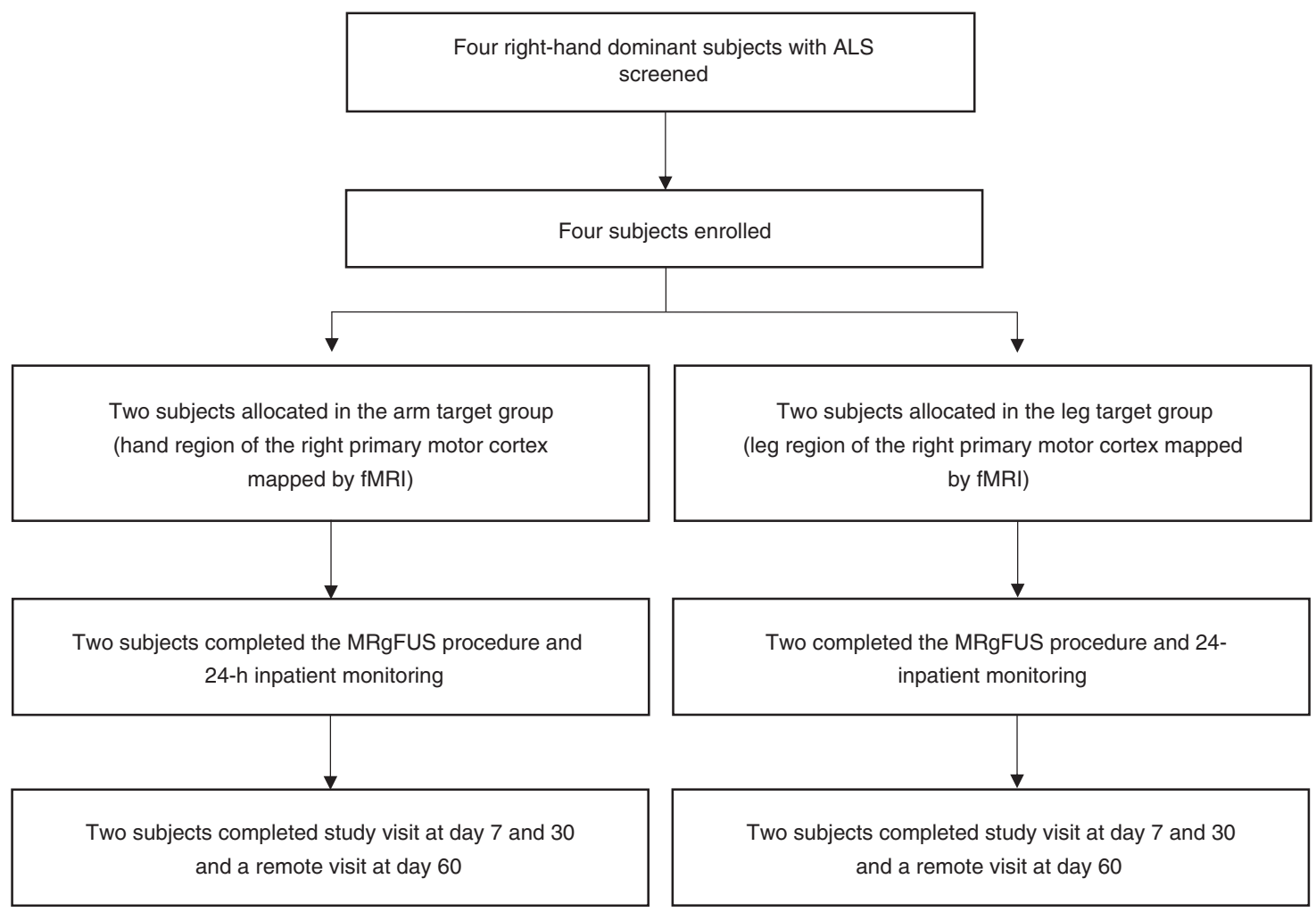

Fig. 1 Trial profile

\begin{tabular}{|c|c|c|c|c|c|c|c|c|c|}
\hline $\begin{array}{l}\text { Disease } \\
\text { duration } \\
\text { (years) }\end{array}$ & $\begin{array}{l}\text { Site of ALS } \\
\text { onset }^{\text {a }}\end{array}$ & $\begin{array}{l}\text { MRC sum } \\
\text { score: } \\
\text { Arms }^{b}\end{array}$ & $\begin{array}{l}\text { MRC sum } \\
\text { score: } \\
\text { Legs }^{b}\end{array}$ & svcc & $\begin{array}{l}\text { ALSFRS-R } \\
\text { score } \\
\text { (total 48) }\end{array}$ & $\begin{array}{l}\text { Modified } \\
\text { Ashworth } \\
\text { score }^{d}\end{array}$ & $\begin{array}{l}\text { MoCA } \\
\text { score } \\
\text { (total 30) }\end{array}$ & $\begin{array}{l}\text { Pre-existing } \\
\text { conditions }\end{array}$ & $\begin{array}{l}\text { ALS } \\
\text { specific } \\
\text { treatment }\end{array}$ \\
\hline 4.2 & $\begin{array}{l}\text { Lower } \\
\text { extremity }\end{array}$ & 52 & 19 & $83 \%$ & 32 & 0 & 26 & $\begin{array}{l}\text { HTN, IBS, GERD, } \\
\text { chronic pain, } \\
\text { osteoporosis, anxiety }\end{array}$ & - \\
\hline 4.5 & $\begin{array}{l}\text { Upper } \\
\text { extremity }\end{array}$ & 27 & 60 & $80 \%$ & 35 & 0 & 27 & HTN, psoriasis & \multirow{2}{*}{$\begin{array}{l}\text { Riluzole and } \\
\text { Edaravone } \\
\text { - }\end{array}$} \\
\hline 4.1 & $\begin{array}{l}\text { Lower } \\
\text { extremity }\end{array}$ & 56 & 16 & $69 \%$ & 33 & 0 & 25 & Chronic pain & \\
\hline $\begin{array}{l}\text { rst reported we } \\
\text { earch Council } \\
\text { er and thumb } \\
\text { pacity (SVC) } \\
\text { es no spasticity } \\
\text { ised ALS Funct } \\
\text { geal reflux dise }\end{array}$ & $\begin{array}{l}\text { is } \\
\text { manual muscl } \\
\text { tors, hip flexors } \\
\text { ported as perc }\end{array}$ & $\begin{array}{l}\text { ength rating } f \\
\text { abductors, } k \\
\text { e of the prec }\end{array}$ & $\begin{array}{l}\text { teral shoulde } \\
\text { ktensors, kne } \\
\text { for age, sex }\end{array}$ & $\begin{array}{l}\text { flexors, } \\
\text { id body }\end{array}$ & $\begin{array}{l}\text { elbow flexors, } \\
\text { ikle dorsiflexors } \\
\text { lass index }\end{array}$ & $\begin{array}{l}\text { w extensors, } \\
\text { plantar flexc }\end{array}$ & $\begin{array}{l}\text { r extensors, } \\
\text { Normal MRC }\end{array}$ & $\begin{array}{l}\mathrm{mb} \text { flexor at the interphalange } \\
\mathrm{n} \text { score is } 70 \text { for both arms ar }\end{array}$ & $\begin{array}{l}\text { joint, abductor of } \\
60 \text { for both legs } \\
\text { ome, GERD }\end{array}$ \\
\hline
\end{tabular}

sequences (Supplementary Fig. 2). This radiologic finding did not correlate with any new symptoms, neurological signs, or focal electroencephalography (EEG) changes and completely resolved at day-30 MRI. In all participants, follow-up MR sequences did not demonstrate parenchymal or subarachnoid hemorrhage, ischemia, gliosis, or worsened precentral gyrus cortical atrophy (Supplementary Fig. 3) up to 30 days following the intervention.

Longitudinal neurological assessments revealed no accelerated disease progression as measured by clinical assessment and Medical Research Council (MRC) scores for the limb contralateral to the sonicated cortex up to 30 days after sonications and
ALS Functional Rating Scale - Revised (ALSFRS-R) scores up to day 60 (Supplementary Table 3). Finally, there were no clinically significant longitudinal changes in cognition or spasticity as measured by Montreal Cognitive Assessment (MoCA) and Modified Ashworth scores, respectively. EEG revealed no changes from baseline and no epileptiform discharges 30 days following BBB opening. All safety laboratory testing was unremarkable.

\section{Discussion}

Therapeutic access to degenerating neurons and glial cells in the primary motor cortex is essential in the development of disease- 


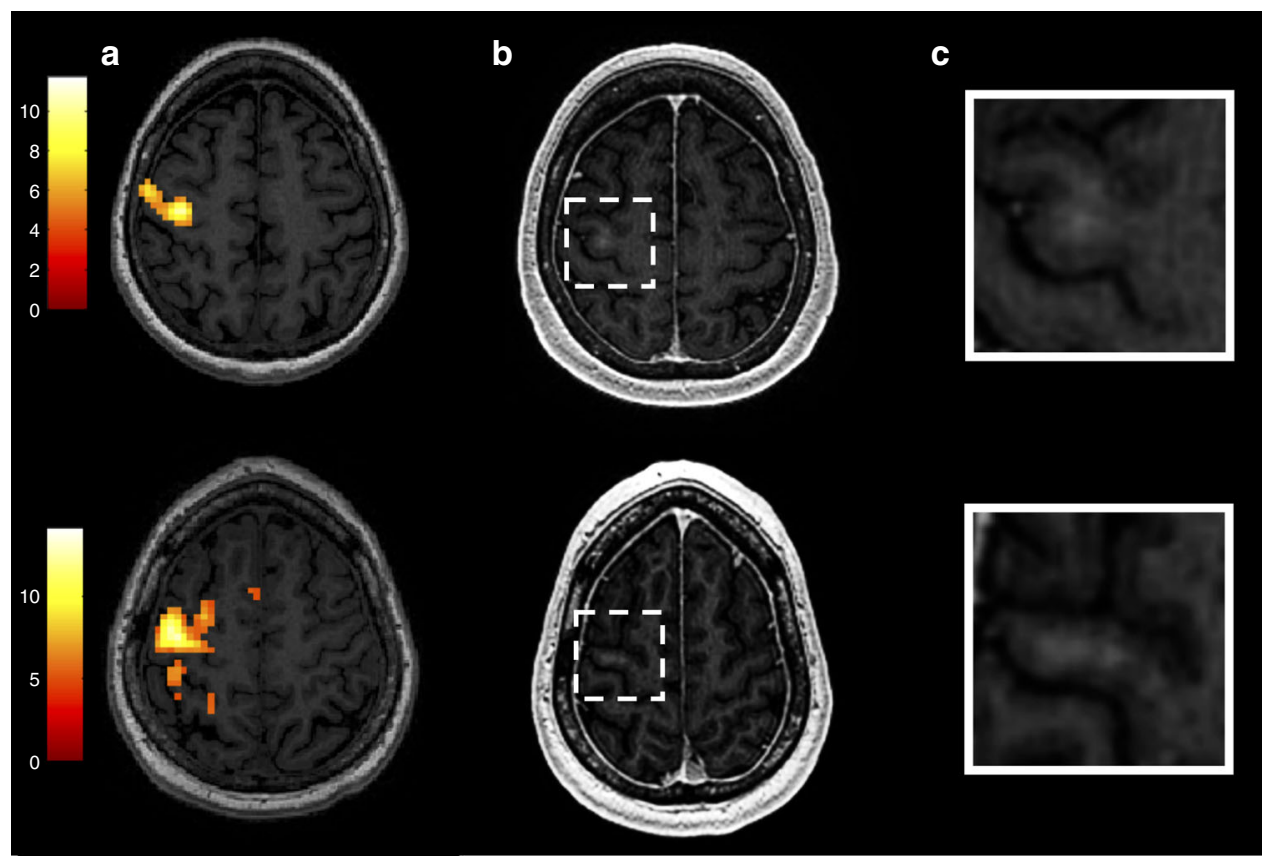

Fig. 2 Feasibility of blood-brain barrier opening using MRgFUS in the hand control region of the right primary motor cortex. a Hand control region mapping using motor-task functional MRI (fMRI). b, c show confirmation of successful blood-brain barrier permeabilization in the sonicated primary motor cortex area as demonstrated by new gadolinium enhancement in T1-weighted imaging. Subject two is represented in the top row and subject three in the bottom row

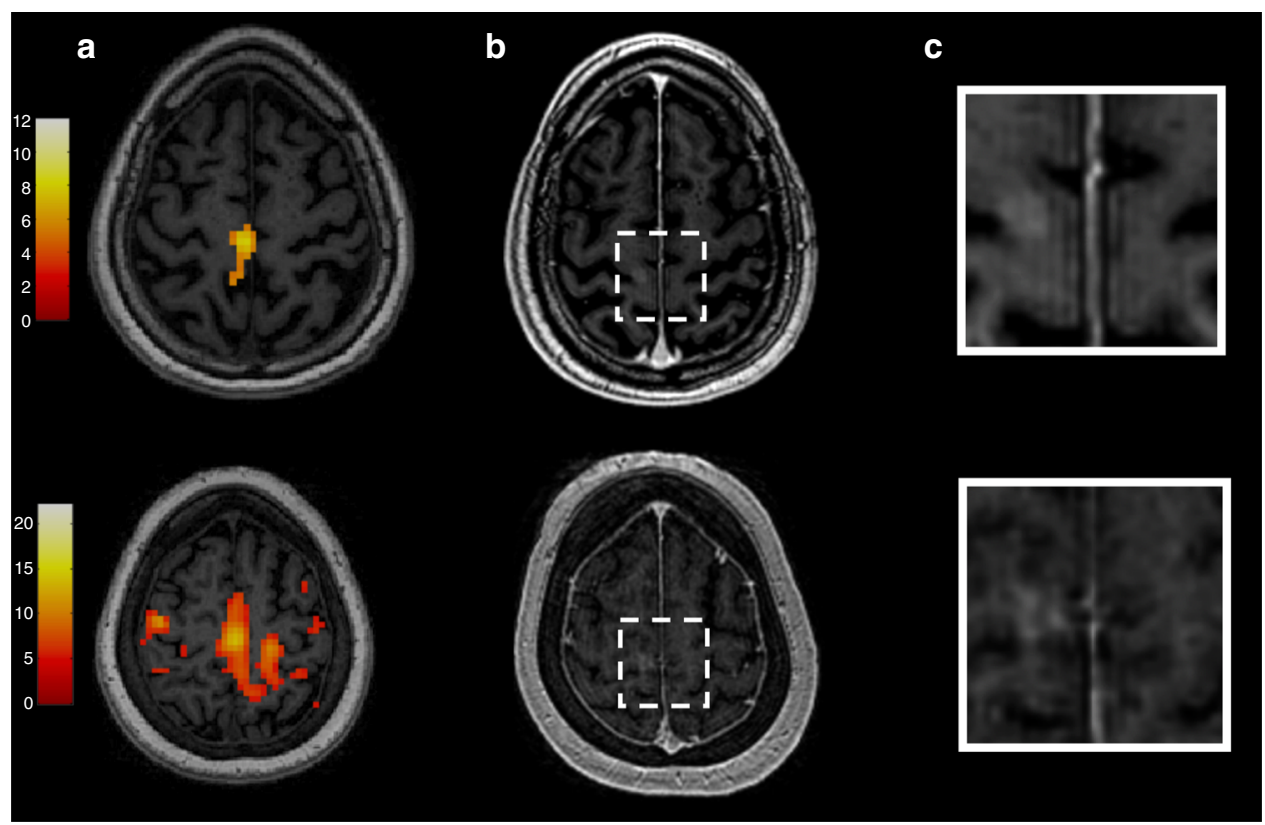

Fig. 3 Feasibility of blood-brain barrier opening using MRgFUS in the leg control region of the right primary motor cortex. a Leg control region mapping using motor-task functional MRI (fMRI). b, c show confirmation of successful blood-brain barrier permeabilization in the sonicated primary motor cortex area as demonstrated by new gadolinium enhancement in T1-weighted imaging. Subject one is represented in the top row and subject four in the bottom row

modifying treatments in ALS. However, the BBB significantly restricts the penetration of numerous interventions from the intravascular compartment to the CNS. In this study, we demonstrated that the BBB can be precisely, safely, and temporarily opened over a targeted region in the primary motor cortex in ALS subjects using transcranial MRgFUS. It also represents the early clinical application of non-invasive, transcranial MRgFUS to safely permeabilize fMRI-directed eloquent brain regions as prior MRgFUS studies targeted sub-cortical white matter regions in humans ${ }^{14,26}$.

Targeting of the primary motor cortex was achieved with millimetre accuracy and tissue sonication was well tolerated by all 


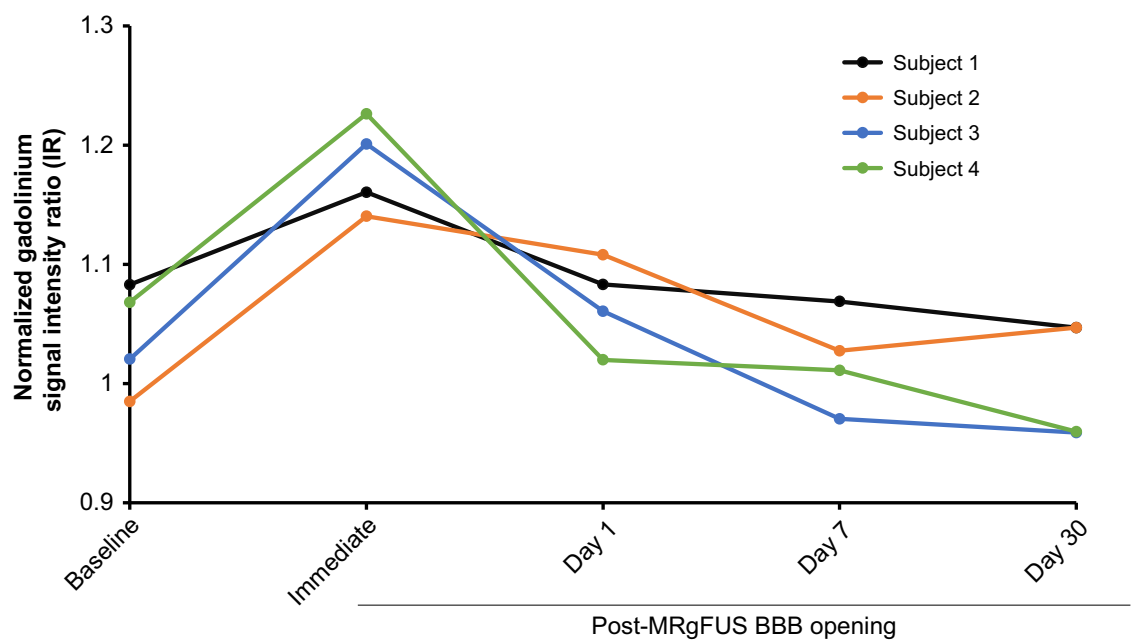

Fig. 4 Baseline and post-procedure gadolinium signal intensity ratio (IR) within the sonicated region on T1-weighted imaging. IRs were normalized to the contralateral, unsonicated mirrored region. Increased IRs were seen immediately after blood-brain barrier opening and returned to baseline within $24 \mathrm{~h}$, indicating reversible BBB permeability. Raw data are provided in the Source Data file

\section{Table 2 Procedure-related adverse events}

\begin{tabular}{llr} 
Adverse event & Severity & N \\
\hline Intraprocedural events & & \\
$\quad$ Headache & Moderate & 3 \\
$\quad$ Vagal response & Moderate & 1 \\
Scalp pain, edema, or bruising attributable to & Mild & 2 \\
placement of the stereotactic frame & & \\
Musculoskeletal pain & Mild to & 2 \\
& moderate & \\
Scalp petechial rash & Mild & 1 \\
Transient FLAIR hyperintensity in sonicated brain & Mild & 1 \\
tissue (Supplementary Fig. 2) & & \\
\hline
\end{tabular}

subjects with no detectable SAEs, hemorrhage or persistent lesions on longitudinal imaging. While GRE is sensitive to microhemorrhages, the absence of changes does not rule out trace erythrocyte extravasation which has been observed in postmortem animal MRgFUS studies ${ }^{18,27}$. In these models, erythrocyte extravasation was generally limited to the perivascular spaces and the animals were asymptomatic. While there is preclinical and post-mortem evidence suggesting endothelial cell dysfunction and impaired BBB repair mechanisms in $\mathrm{ALS}^{8}$, in all participants of this study, BBB permeabilization was reversible, without radiological evidence of significant inflammation or tissue damage 30 days after the procedure. One participant (subject four) developed a delayed and self-limited, small parenchymal FLAIR hyperintensity within the sonicated region. This asymptomatic finding was inconsistent with hemorrhage, ischemia, or gliosis and completely resolved on imaging obtained 30 days following the intervention. While the aetiology and clinical significance are uncertain, the finding may represent serum extravasation of gadolinium, albumin and/or immunoglobulins from BBB opening ${ }^{28,29}$.

The complication risk of the MRgFUS BBB opening procedure is mitigated by the inclusion of a number of key technical and design safety features. First, the optimal sonication power for safe $\mathrm{BBB}$ disruption at each target is determined through tissuespecific ramp test, based on the acoustic and cavitation feedback measured by the MRgFUS system ${ }^{27}$. Second, real-time MR thermography and acoustic spectrum monitoring, along with the patient's ability to communicate adverse symptoms, ensure target accuracy, tissue integrity and prompt recognition of any complication during sonication. These intraprocedural safety assessments are of particular importance when targeting eloquent brain regions with increased vascularity, such as the primary motor cortex. With these safeguards in place, BBB opening with MRgFUS likely poses a lower risk and faster recovery time than open neurosurgical procedures.

MRgFUS-mediated BBB opening is a non-invasive alternative to stereotactic surgery for the targeted delivery of therapeutics. Preclinical studies have demonstrated the effectiveness of MRgFUS in enhancing targeted brain bioavailability of large drugs (e.g., chemotherapeutics ${ }^{23,30}$, antibodies ${ }^{20,31-33}$, AAV921, and cells ${ }^{34-36}$ ) injected intravascularly in animal models. Multiple MRgFUS procedures are also feasible for therapeutics with repeated dosing as demonstrated in a pilot MRgFUS trial in subjects with $\mathrm{AD}^{14}$ and preclinical studies ${ }^{36,37}$ MRgFUS creates a window for candidate large investigational compounds, infused peripherally to target degenerating neurons and glial cells in the primary motor cortex of ALS patients. In ALS animal models, antibodies $^{38,39}$, AAVs $^{3}$ and progenitor cells ${ }^{11}$ were efficacious in prolonging survival, but required invasive surgical delivery procedures.

Antibody-mediated reduction of misfolded or toxic proteins is a therapeutic strategy in $\mathrm{ALS}^{3,39}$. In SOD1 transgenic ALS mice, antibodies binding to misfolded SOD1 were effective in prolonging survival following an intraventricular infusion ${ }^{38,39}$. The technical feasibility and utility of MRgFUS in delivering antibodies to sonicated tissue has been demonstrated in animal models of $\mathrm{AD}$ and brain tumours ${ }^{20,31-33}$. Humanized antibodies to key epitopes such as cortical misfolded SOD1 or targets of neuroinflammation and survival pathways implicated in ALS can be coupled with MRgFUS for brain delivery in future trials ${ }^{14}$.

Effective application of upstream strategies to knock down SOD1 expression using AAV9-mediated RNA therapy requires delivery by ventricular intrathecal injection or surgical implantation in the motor cortex ${ }^{3,40}$. In contrast to intrathecal injections, MRgFUS significantly optimized the targeted AAV9 bioavailability in sonicated brain regions with lower intravenous 
viral load required ${ }^{21}$. Also, while AAV9 introduced via lumbosacral intrathecal administration can diffuse across multiple anterior horn levels of the spinal cord, its diffusion into the motor cortex was limited ${ }^{41}$, highlighting an additional advantage of the MRgFUS-mediated brain delivery.

Finally, another promising strategy in preclinical ALS models is to enhance neurotrophic support in the motor cortex. Thomsen et al. ${ }^{11}$ demonstrated increased neuroprotective effects of glial cell line-derived neurotrophic factor (GDNF) after stereotactic intracortical transplantation of GDNF-secreting neural progenitor cells, leading to significant UMN and LMN protection. MRgFUS has been safely used to deliver progenitor cells ${ }^{34}$ and other cell types ${ }^{36}$ to discrete brain regions in animal models following intra-arterial and intravenous cellular injection and may be a non-invasive delivery option in future studies.

To enhance a potential synergistic effect on UMNs and LMNs, targeted brain therapeutic delivery using MRgFUS can be coupled with non-targeted, lumbosacral intrathecal therapeutic injections, which have unreliable motor cortex penetration and are currently being used in ALS clinical trials. MRgFUS blood-spinal cord barrier opening has not yet been developed in humans, but has been performed safely in the spinal cord of non-ALS rodent models ${ }^{42,43}$. An early study demonstrated enhanced chemotherapy delivery using MRgFUS blood-spinal cord barrier opening in rodents ${ }^{44}$. However, the bony vertebrae of the spinal column in primates present a technical challenge for focusing the ultrasound and further validation and optimization studies are necessary before translation to humans ${ }^{45}$.

This study represents the initial step towards the validation of a non-invasive brain delivery platform in ALS, but there are several limitations. First, the ALS cohort enrolled in this open-label, single-centre study was small with limited generalizability and power to infer AE rates with precision. For safety concerns, only patients with limb-onset ALS and severe weakness were included and only a small volume of the primary motor cortex was targeted. While the minimal volume of BBB opening for sufficient therapeutic distribution is unknown and likely depends on drug properties, future trials will explore the safety and feasibility of sonicating larger volumes or the entirety of the primary motor cortex, along with frontotemporal regions that are also affected in ALS with frontotemporal dementia. Current preclinical evidence support the feasibility of targeting large hemispheric volumes in canines, whose brain dimensions more closely resemble that of humans ${ }^{46}$. Further, the clinical MRgFUS device can achieve a larger volume of BBB opening through a summation of targets and wider spacing of spots within each target ${ }^{14}$. Ongoing clinical trials using focused ultrasound (NCT03616860, NCT03712293, NCT03714243, NCT03626896) as well as pulsed ultrasound (NCT03744026) are underway to treat larger tumour regions and peri-tumour tissue to enhance chemotherapy delivery.

The qualitative and semi-quantitative assessment of BBB permeability through contrast T1-weighted MRI is also a limitation in this study. Because the peak gadolinium enhancement is expected to be immediately after BBB opening, a delay between sonications and contrast MRI acquisition due to patient transfer, head frame removal, and rest will contribute to decreased visualized IR in the sonicated target. In future MRgFUS BBB opening trials, dynamic-contrast enhanced MRI sequence and MR coil improvements can more quantitatively and accurately assess BBB permeability.

Cortical plasticity and functional reorganization of the motor homunculus topography in ALS ${ }^{47,48}$ can limit the ascertainment of functional regions of the primary motor cortex. We found motor activation by fMRI was necessary in most of the subjects to more accurately determine the functional arm and leg-controlling cortical regions. We observed lateral shifts in the cortical hand area, as well as overactivation of bi-hemispheric anterior structures (e.g. premotor and supplementary motor area) as previously described $^{47-49}$.

In addition to providing a window for therapeutic access, BBB opening exposes the central nervous system microenvironment to circulating proteins. For instance, transient microglial activation was observed in a transgenic rodent model of $\mathrm{AD}$ after $\mathrm{BBB}$ opening and resolved at 15 days $^{50}$. The implication of transient microglial activation in ALS is unknown as humoral and cellular mechanisms have been associated with both neuroprotection and toxicity ${ }^{51}$. Neuroinflammation and endothelial, neuronal and glial function should be further investigated in upcoming MRgFUS trials using advanced MR imaging and spectroscopy, cortical excitability measures (e.g., short intracortical interval parameter of threshold-tracking transcranial magnetic stimulation), PET imaging (e.g. TPSO $^{52}$ ), along with serum and cerebrospinal fluid biomarkers.

In conclusion, the reversible permeabilization of the $\mathrm{BBB}$ in small regions of the primary motor cortex using transcranial MRgFUS with microbubbles was safe and tolerated in subjects with ALS. This study represents the seminal initial step in establishing a delivery platform whereby MRgFUS-mediated BBB opening can be coupled with the systemic administration of the most promising ALS therapeutics to directly target the degenerating motor cortex.

\section{Methods}

Study design. This prospective, open-label, single-arm, single-centre first-inhuman study was designed to evaluate the technical feasibility, reversibility and safety of BBB opening in the primary motor cortex (precentral gyrus) of subjects with ALS. The non-dominant motor cortex region corresponding to a severely weakened contralateral limb was chosen. The study was approved by the Research Ethics Board at Sunnybrook Health Sciences Centre (SHSC) and Health Canada and was registered with ClinicalTrials.gov NCT03321487. All subjects provided written informed consent prior to enrolment after a detailed discussion of the study rationale, risks and the investigational nature of the procedure. This trial complied with the International Conference on Harmonization guideline for Good Clinical Practice, Tri-Council Policy Statement on ethical conduct for research involving humans (TCPS-2), and ISO 14155

Participants. Four right-hand dominant participants aged $\geq 18$ years were enrolled in the study. Each was diagnosed with laboratory-supported probable, clinically probable or definite ALS according to the revised El Escorial criteria ${ }^{53}$, which requires evidence of UMN dysfunction in at least one body region. Participants had slow vital capacity (SVC) $\geq 50 \%$ for predicted age and body habitus and severe left hand or leg weakness as defined by MRC muscle strength grade $\leq 3$ in index finger abduction and thumb abduction or hip flexors and ankle dorsiflexors, respectively. Participants on a stable dose of oral riluzole (up to $50 \mathrm{mg}$ twice daily) for at leas 30 days or intravenous edaravone for at least two cycles were eligible for the study. Edaravone and riluzole were not administered on the day of the MRgFUS procedure and restarted after discharge. Efforts were made to balance sexes in this study

Important exclusion criteria included co-existent frontotemporal dementia, systemic or cerebral vasculopathy, auto-immune conditions, bleeding disorder or taking an anticoagulant, contraindication to MRI or gadolinium contrast, intracranial hemorrhage or prohibitive structural lesions seen on baseline MRI, American Society of Anesthesiologist classification > III, cystatin C glomerular filtration rate $<30 \mathrm{~mL}$ per min per $1.73 \mathrm{~m}^{2}$, unstable cardiovascular or pulmonary disease, or contraindication to intravenous microbubble injection, including known or suspected cardiac shunt or perflutren hypersensitivity. Urine pregnancy test and use of contraception during the course of the study was required for women of childbearing potential. Detailed eligibility criteria are listed in Supplementary Methods.

Enrolled patients were allocated in an open-label manner to the arm $(n=2)$ or leg $(n=2)$ target group depending on which left-sided limb was weaker. In each group, MRgFUS sonications targeted the right motor homunculus region corresponding to the hand or leg and aimed to open a maximum BBB volume of 1 $\mathrm{cm}^{3}$. Target accuracy was ascertained using hand or foot task activation functional MRI prior to the sonication as described below.

Functional MRI acquisition and task. All fMRIs were performed using the same study 3-Tesla scanner (Signa MR750; GE Healthcare, Milwaukee, Wis.) with an eight-channel head receiver coil. Anatomical images were acquired with $3 \mathrm{D}$ fast spoiled gradient echo (3D-FSPGR) [IR-FSPGR (inversion recovery prepared fast spoiled gradient echo)] sequence with $1 \mathrm{~mm}$ thickness. The scan parameters were 
$\mathrm{TE}=2.94 \mathrm{~ms}, \mathrm{TR}=7.65 \mathrm{~ms}$, and matrix size $=265 \times 265 . \mathrm{fMRI}$ parameters were 130 temporal volumes of 36 slices with $4 \mathrm{~mm}$ thickness; TR $=2000 \mathrm{~ms}$, TE $=30$ $\mathrm{ms}$; flip angle $=70^{\circ}$; and matrix size $=64 \times 64$. The first four volumes were discarded for $\mathrm{T} 1$ effects. The functional activation images were acquired during a block-design motor task with total duration of 4 min $28 \mathrm{~s}$. Six blocks of repetitive movements to written command were interspersed with blocks of black-on-white crosshair fixation to elicit the blood-oxygen-level-dependent hemodynamic response to motor activity. Each block lasted for ten seconds. Subjects were instructed prior to entering the scanner to perform hand squeezes or foot taps and had the opportunity to practice.

MR-guided focused ultrasound intervention. The MRgFUS BBB opening procedure was performed using the $220 \mathrm{kHz}$ ExAblate Neuro 4000 system type 2.0 (InSightec ${ }^{\circledast}$, Israel) with 1024 ultrasound transducers coupled to the 3-Tesla MRI. First, the subject's hair was closely shaved to the scalp. For stereotactic accuracy, each subject was fitted with a Cosman-Roberts-Wells stereotactic frame under local anaesthetic. Each subject was placed supine on the MR table with compression stockings and care was taken to pad all pressure points. The frame was then fixed to the ExAblate helmet with intervening degassed water between the scalp and transducers.

An anaesthesiologist was present for the entire procedure to monitor vital signs and to provide analgesia and conscious sedation as necessary. MR-compatible, non-invasive monitoring of vital signs and electrocardiogram was maintained throughout the procedure. The subjects remained awake and had available a stop mechanism in case of discomfort or emergency. First, planning MR sequences were acquired for registration to baseline CT scan. To achieve BBB opening within the arm or leg regions, two sonication targets of $5 \times 5 \times 7 \mathrm{~mm}\left(175 \mathrm{~mm}^{3}\right.$ each, 350 $\mathrm{mm}^{3}$ total) were placed over the arm or leg region as mapped by task fMRI, avoiding overlap with any voxels containing sulci and visible vessels within two contiguous axial slices as to mitigate the potential risk of hemorrhage. Within each sonication target, the focused ultrasound beam was electronically steered among four spots within the target as represented in Supplementary Fig. 4A.

For transcranial focused ultrasound applications, accurate estimation of in vivo tissue pressure for individual subjects at this level of power is limited given highly variable skull geometry, density, and target locations. Therefore, the optimal power for BBB opening was determined by cavitation feedback at each target through a ramp test ${ }^{14,27}$. This involves applying short sonications with $5 \%$ power increments until the device's hydrophones detect sub-harmonic acoustic feedback from the target, indicating the cavitation threshold. The optimal power for BBB opening was estimated as $50 \%$ of this threshold.

The power ramp test was then followed by one to two $90 \mathrm{~s}$ sonication cycles until the BBB was opened, each coupled with intravenous $4 \mu \mathrm{l} / \mathrm{kg}$ perflutren microbubbles (Definity ${ }^{\circledR}$, Lantheus Medical Imaging, USA). The total dose of perflutren did not exceed $20 \mu \mathrm{l} / \mathrm{kg}$. During each $90 \mathrm{~s}$ sonication cycle, ultrasound was delivered in burst mode with pulse repetition period of $300 \mathrm{~ms}$ and $0.88 \%$ duty cycle per each of the four spots within a target (Supplementary Fig. 4B).

Real-time monitoring during sonications included acoustic monitoring, MR thermometry, and direct patient feedback. We were able to acquire MR thermometry without interference, using standard fast spoiled gradient echo (FSPGR) sequence with parameters $\mathrm{TR}=26 \mathrm{~ms}$ and $\mathrm{TE}=13 \mathrm{~ms}$. Conversely, there is no MR interference to cavitation receivers built-in the focused ultrasound array. Subsequent to each sonication cycle, the subject was examined clinically and radiologically for any AEs, including gadolinium enhanced T1-weighted and GRE sequences to assess BBB permeability, tissue integrity and microhemorrhage. Visualization of new gadolinium enhancement in the sonicated brain parenchyma targeted signified successful BBB opening and the procedure was terminated. Subjects then returned for MRI with the head coil for high definition imaging. All subjects were admitted to the neurosurgical unit for overnight observation followed by clinical and MRI assessments for reversibility of BBB opening and AEs before discharge from hospital the next day.

Outcomes. The primary outcome was safety and feasibility of transient BBB opening by MRgFUS in the primary motor cortex. Safety was measured as the occurrence and severity of device- and procedure-related AEs and SAEs over the 60 -day study duration. AE and SAE definitions are detailed in Supplementary Note 1 and Supplementary Table 4 and were captured by clinical assessment, laboratory testing, and neuroimaging immediately after BBB opening, and at day 1 , 7 , and 30 post-MRgFUS procedure and over the phone at day 60 . Neurological exam included MRC manual muscle strength rating (grade 0-5) of the shoulder extensors, elbow flexors, elbow extensors, finger extensors, thumb flexor at the interphalangeal joint, abductor of the index finger, thumb abductors, hip flexors, hip abductors, knee extensors, knee flexors, ankle dorsiflexors and plantar flexors. Normal MRC sum score for the arm is 35 and for the leg is 30 . Safety laboratory testing included complete blood count, C-reactive protein, erythrocyte sedimentation rate, creatinine, cystatin-C, liver enzymes and electrolytes. Safety MRI sequences included T1 with and without gadolinium, T2, FLAIR, GRE, and DWI.

Feasibility was qualitatively defined as detectable gadolinium enhancement signal into the sonicated targets on T1-weighted imaging immediately post-procedure, and resolution of the enhancement by the next day (reversibility criterion). This was further quantified by the gadolinium signal intensity ratio (IR) within a region of interest (ROI) in the targeted area, normalized to the contralateral, unsonicated mirrored ROI. Square ROIs were created the size of and centred on the sonication targets, which are illustrated in Fig. S1. Exploratory measures (modified Ashworth Scale for spasticity, MoCA, ALSFRS-R, and EEG) were collected at baseline and at day 30. The final study event was a remote visit 60 days after the procedure to collect AEs and ALSFRS-R. In-person and remotely collected ALSFRS-R scores have excellent and comparable inter- and intra-rater reliability ${ }^{54}$.

Data analysis. Aggregate data was reported as median and range for continuous variables and percentages for categorical variables. MR enhancement from gadolinium leakage post-sonication was used as a surrogate marker of BBB permeability, and this was quantified by the signal IR longitudinally. Task fMRIs were analysed using Statistical Parametric Model (SPM) version 12. Briefly, preprocessing consisted of motion correct, slice-time correction, functional realignment, co-registration to anatomic image, structural segmentation, functional and structural normalization, and spatial smoothing with $6 \mathrm{~mm}$ Gaussian kernel. The activation measure of interest was contrast of movement greater than fixation. The individual statistical parametric maps were generated from t-statistics after family wise error corrected $p<0.05$, which was then overlaid on the patient specific structural scans to identify the gyrus activated by the motor task.

In order to assess the precentral gyrus cortical volume over time, we processed the structural T1-weighted MRIs with the longitudinal stream in Freesurfer surface-based analysis ${ }^{55}$, which is documented and available online (http://surfer. nmr.mgh.harvard.edu/). The longitudinal stream increases the reliability of within subject estimates by creating unbiased within-subject template space and image on which processing steps (e.g. skull stripping, atlas transforms and registration, and parcellations) are then performed.

Reporting summary. Further information on research design is available in the Nature Research Reporting Summary linked to this article.

\section{Data availability}

The authors declare that all the data supporting the findings of this study are available within the paper and its supplementary information files. Study protocol, de-identified individual-participant data and data dictionary are available from the corresponding author upon request.

Received: 8 February 2019; Accepted: 6 September 2019; Published online: 26 September 2019

\section{References}

1. Dadon-Nachum, M., Melamed, E. \& Offen, D. The 'dying-back' phenomenon of motor neurons in ALS. J. Mol. Neurosci. MN 43, 470-477 (2011).

2. Eisen, A. et al. Cortical influences drive amyotrophic lateral sclerosis. J. Neurol. Neurosurg. Psychiatry 88, 917-924 (2017).

3. Thomsen, G. M. et al. Delayed disease onset and extended survival in the SOD1G93A rat model of amyotrophic lateral sclerosis after suppression of mutant SOD1 in the motor cortex. J. Neurosci. 34, 15587-15600 (2014).

4. Handley, E. E. et al. Synapse dysfunction of layer V pyramidal neurons precedes neurodegeneration in a mouse model of TDP-43 proteinopathies. Cereb. Cortex 27, 3630-3647 (2017).

5. Ng, M.-C. et al. Abnormal diffusion tensor in nonsymptomatic familial amyotrophic lateral sclerosis with a causative superoxide dismutase 1 mutation. J. Magn. Reson. Imaging 27, 8-13 (2007).

6. Menon, P., Kiernan, M. C. \& Vucic, S. Cortical hyperexcitability precedes lower motor neuron dysfunction in ALS. Clin. Neurophysiol. 126, 803-809 (2015).

7. Garbuzova-Davis, S. et al. Ultrastructure of blood-brain barrier and bloodspinal cord barrier in SOD1 mice modeling ALS. Brain Res. 1157, 126-137 (2007).

8. Garbuzova-Davis, S. \& Sanberg, P. R. Blood-CNS barrier impairment in ALS patients versus an animal model. Front Cell Neurosci. 8, 21 (2014).

9. Thomson, A. et al. Potential new complication in drug therapy development for amyotrophic lateral sclerosis. Expert Rev. Neurother. 16, 1397-1405 (2016).

10. Jablonski, M. R. et al. Inhibiting drug efflux transporters improves efficacy of ALS therapeutics. Ann. Clin. Transl. Neurol. 1, 996-1005 (2014).

11. Thomsen, G. M. et al. Transplantation of neural progenitor cells expressing glial cell line-derived neurotrophic factor into the motor cortex as a strategy to treat amyotrophic lateral sclerosis. Stem Cells 36, 1122-1131 (2018).

12. Martinez, H. R. et al. Stem-cell transplantation into the frontal motor cortex in amyotrophic lateral sclerosis patients. Cytotherapy 11, 26-34 (2009).

13. Boado, R. J., Hui, E. K.-W., Lu, J. Z., Zhou, Q.-H. \& Pardridge, W. M. Reversal of lysosomal storage in brain of adult MPS-I mice with intravenous trojan horse-iduronidase fusion protein. Mol. Pharm. 8, 1342-1350 (2011). 
14. Lipsman, N. et al. Blood-brain barrier opening in Alzheimer's disease using MR-guided focused ultrasound. Nat. Commun. 9, 2336 (2018).

15. Hynynen, K. \& Jolesz, F. A. Demonstration of potential noninvasive ultrasound brain therapy through an intact skull. Ultrasound Med. Biol. 24, 275-283 (1998).

16. Elias, W. J. et al. A randomized trial of focused ultrasound thalamotomy for essential tremor. N. Engl. J. Med. 375, 730-739 (2016).

17. Hynynen, K., McDannold, N., Vykhodtseva, N., Jolesz, F. A. \& Noninvasive, M. R. imaging-guided focal opening of the blood-brain barrier in rabbits. Radiology 220, 640-646 (2001)

18. McDannold, N., Vykhodtseva, N., Raymond, S., Jolesz, F. A. \& Hynynen, K. MRI-guided targeted blood-brain barrier disruption with focused ultrasound: histological findings in rabbits. Ultrasound Med. Biol. 31, 1527-1537 (2005)

19. McDannold, N., Arvanitis, C. D., Vykhodtseva, N. \& Livingstone, M. S. Temporary disruption of the blood-brain barrier by use of ultrasound and microbubbles: safety and efficacy evaluation in rhesus macaques. Cancer Res. 72, 3652-3663 (2012).

20. Jordão, J. F. et al. Antibodies targeted to the brain with image-guided focused ultrasound reduces amyloid-beta plaque load in the TgCRND8 mouse model of Alzheimer's disease. PLoS ONE 5, e10549 (2010).

21. Thévenot, E. et al. Targeted delivery of self-complementary adeno-associated virus serotype 9 to the brain, using magnetic resonance imaging-guided focused ultrasound. Hum. Gene Ther. 23, 1144-1155 (2012).

22. Noroozian, Z. et al. MRI-guided focused ultrasound for targeted delivery of raav to the brain. Methods Mol. Biol. 1950, 177-197 (2019).

23. Alli, S. et al. Brainstem blood brain barrier disruption using focused ultrasound: a demonstration of feasibility and enhanced doxorubicin delivery. J. Control Release 281, 29-41 (2018).

24. Carpentier, A. et al. Clinical trial of blood-brain barrier disruption by pulsed ultrasound. Sci. Transl. Med. 8, 343re2 (2016).

25. Idbaih, A. et al. Safety and feasibility of repeated and transient blood-brain barrier disruption by pulsed ultrasound in patients with recurrent glioblastoma. Clin. Cancer Res. https://doi.org/10.1158/1078-0432.CCR-183643 (2019)

26. Mainprize, T. et al. Blood-brain barrier opening in primary brain tumors with non-invasive MR-guided focused. Ultrasound.: A Clin. Saf. Feasibility Study Sci. Rep. 9, 321 (2019).

27. Huang, Y., Alkins, R., Schwartz, M. L. \& Hynynen, K. Opening the bloodbrain barrier with MR imaging-guided focused ultrasound: preclinical testing on a trans-human skull porcine model. Radiology 282, 123-130 (2017).

28. Kovacs, Z. I. et al. Disrupting the blood-brain barrier by focused ultrasound induces sterile inflammation. Proc. Natl Acad. Sci. USA. 114, E75-E84 (2017).

29. Silburt, J., Lipsman, N. \& Aubert, I. Disrupting the blood-brain barrier with focused ultrasound: Perspectives on inflammation and regeneration. Proc. Natl Acad. Sci. USA 114, E6735-E6736 (2017).

30. Coluccia, D. et al. Enhancing glioblastoma treatment using cisplatin-goldnanoparticle conjugates and targeted delivery with magnetic resonance-guided focused ultrasound. Nanomed 14, 1137-1148 (2018).

31. Kobus, T., Zervantonakis, I. K., Zhang, Y. \& McDannold, N. J. Growth inhibition in a brain metastasis model by antibody delivery using focused ultrasound-mediated blood-brain barrier disruption. J. Control. Release . J. Control. Release Soc. 238, 281-288 (2016).

32. Liu, H.-L. et al. Focused ultrasound enhances central nervous system delivery of bevacizumab for malignant glioma treatment. Radiology 281, 99-108 (2016).

33. Alecou, T., Giannakou, M. \& Damianou, C. Amyloid $\beta$ plaque reduction with antibodies crossing the blood-brain barrier, which was opened in 3 sessions of focused ultrasound in a rabbit model. J. Ultrasound Med. 36, 2257-2270 (2017).

34. Burgess, A. et al. Targeted delivery of neural stem cells to the brain using MRIguided focused ultrasound to disrupt the blood-brain barrier. PLoS One 6, e27877 (2011)

35. Alkins, R. et al. Focused ultrasound delivers targeted immune cells to metastatic brain tumors. Cancer Res. 73, 1892-1899 (2013)

36. Alkins, R., Burgess, A., Kerbel, R., Wels, W. S. \& Hynynen, K. Early treatment of HER2-amplified brain tumors with targeted NK-92 cells and focused ultrasound improves survival. Neuro-Oncol. 18, 974-981 (2016).

37. Nisbet, R. M. et al. Combined effects of scanning ultrasound and a tau-specific single chain antibody in a tau transgenic mouse model. Brain J. Neurol. 140, 1220-1230 (2017).

38. Urushitani, M., Ezzi, S. A. \& Julien, J.-P. Therapeutic effects of immunization with mutant superoxide dismutase in mice models of amyotrophic lateral sclerosis. Proc. Natl Acad. Sci. USA 104, 2495-2500 (2007).

39. Gros-Louis, F., Soucy, G., Lariviere, R. \& Julien, J.-P. Intracerebroventricular infusion of monoclonal antibody or its derived Fab fragment against misfolded forms of SOD1 mutant delays mortality in a mouse model of ALS. J. Neurochem. 113, 1188-1199 (2010).
40. Stoica, L. et al. Adeno-associated virus-delivered artificial microRNA extends survival and delays paralysis in an amyotrophic lateral sclerosis mouse model. Ann. Neurol. 79, 687-700 (2016).

41. Wang, H. et al. Widespread spinal cord transduction by intrathecal injection of rAAV delivers efficacious RNAi therapy for amyotrophic lateral sclerosis. Hum. Mol. Genet. 23, 668-681 (2014).

42. Weber-Adrian, D. et al. Gene delivery to the spinal cord using MRI-guided focused ultrasound. Gene Ther. 22, 568-577 (2015).

43. Payne, A. H. et al. Magnetic resonance imaging-guided focused ultrasound to increase localized blood-spinal cord barrier permeability. Neural Regen. Res. 12, 2045-2049 (2017).

44. O'Reilly, M. A. et al. Preliminary investigation of focused ultrasoundfacilitated drug delivery for the treatment of leptomeningeal metastases. Sci. Rep. 8, 9013 (2018).

45. Fletcher, S. P. \& O’Reilly, M. A. Analysis of multifrequency and phase keying strategies for focusing ultrasound to the human vertebral canal. IEEE Trans. Ultrason. Ferroelectr. Freq. Control 65, 2322-2331 (2018).

46. O'Reilly, M. A. et al. Investigation of the safety of focused ultrasound-induced blood-brain barrier opening in a natural canine model of aging. Theranostics 7, 3573-3584 (2017).

47. Konrad, C. et al. Pattern of cortical reorganization in amyotrophic lateral sclerosis: a functional magnetic resonance imaging study. Exp. Brain Res. 143, 51-56 (2002)

48. Lule, D. et al. Cortical plasticity in amyotrophic lateral sclerosis: motor imagery and function. Neurorehabil. Neural Repair 21, 518-526 (2007).

49. Stanton, B. R. et al. Altered cortical activation during a motor task in ALS. Evidence for involvement of central pathways. J. Neurol. 254, 1260-1267 (2007).

50. Jordão, J. F. et al. Amyloid- $\beta$ plaque reduction, endogenous antibody delivery and glial activation by brain-targeted, transcranial focused ultrasound. Exp. Neurol. 248, 16-29 (2013).

51. Thonhoff, J. R., Simpson, E. P. \& Appel, S. H. Neuroinflammatory mechanisms in amyotrophic lateral sclerosis pathogenesis. Curr. Opin. Neurol. 31, 635-639 (2018).

52. Albrecht, D. S., Granziera, C., Hooker, J. M. \& Loggia, M. L. In vivo imaging of human neuroinflammation. ACS Chem. Neurosci. 7, 470-483 (2016).

53. Brooks, B. R., Miller, R. G., Swash, M. \& Munsat, T. L. El Escorial revisited: revised criteria for the diagnosis of amyotrophic lateral sclerosis. Amyotroph. Lateral Scler. Other Mot. Neuron Disord. 1, 293-299 (2000).

54. Kaufmann, P. et al. Excellent inter-rater, intra-rater, and telephoneadministered reliability of the ALSFRS-R in a multicenter clinical trial. Amyotroph. Lateral Scler. 8, 42-46 (2007).

55. Reuter, M., Schmansky, N. J., Rosas, H. D. \& Fischl, B. Within-subject template estimation for unbiased longitudinal image analysis. Neuroimage $\mathbf{6 1}$, 1402-1418 (2012).

\section{Acknowledgements}

This investigator-initiated study was funded by an ALS Society of Canada peer-reviewed grant and generosity of philanthropic gifts to the Sunnybrook Foundation by the Temerty, Stock, Lechem and Harquail families. The authors are grateful to InSightec ${ }^{\oplus}$ Ltd (Israel) for the regulatory and technical sponsorship throughout the study. Both InSightec $^{\oplus}$ and the ALS Society of Canada had no role in study design, data collection, analysis, interpretation or reporting. The authors also would like to thank Ruby Endre and Garry Detzler for their technical support in the study. N.L. gratefully acknowledge the philanthropic support provided through the Sunnybrook Foundation and the Harquail Centre for Neuromodulation. Finally, we are grateful to the patients and their families for their involvement and contributions.

\section{Author contributions}

A.A., K.H., T.M., S.E.B., I.A., N.L., and L.Z. contributed to the study conceptualization and design. A.A., Y.M., C.Hamani, M.L., C.Heyn, N.L., and L.Z. participated in data collection. Y.H. and K.H. provided technical focused ultrasound expertise. A.A., Y.M., N. L., and L.Z. had full access to the data and contributed to the analysis and interpretation. A.A. and Y.M. served as co-first authors; N.L. and L.Z. served as co-senior authors of the paper. All authors critically revised the paper.

\section{Competing interests}

N.L., K.H., and S.E.B. have received an honorarium from the Focused Ultrasound Foundation for serving on an expert steering committee on focused ultrasound in $\mathrm{Alz}$ heimer's Disease. K.H. is an inventor on intellectual property owned by Brigham and Women's hospital in Boston and Sunnybrook Research Institute in Toronto related to intracranial focused ultrasound technology. A.A., Y.M., M.L., C.Hamani, Y.H., T.M., I.A., C.Heyn, N.L., and L.Z. declare no competing interests. 


\section{Additional information}

Supplementary information is available for this paper at https://doi.org/10.1038/s41467019-12426-9.

Correspondence and requests for materials should be addressed to A.A.

Peer review information Nature Communications thanks Alexandre Carpentier and Andrea Malaspina for their contribution to the peer review of this work. Peer reviewer reports are available.

Reprints and permission information is available at http://www.nature.com/reprints

Publisher's note Springer Nature remains neutral with regard to jurisdictional claims in published maps and institutional affiliations. (c) (i) Open Access This article is licensed under a Creative Commons Attribution 4.0 International License, which permits use, sharing, adaptation, distribution and reproduction in any medium or format, as long as you give appropriate credit to the original author(s) and the source, provide a link to the Creative Commons license, and indicate if changes were made. The images or other third party material in this article are included in the article's Creative Commons license, unless indicated otherwise in a credit line to the material. If material is not included in the article's Creative Commons license and your intended use is not permitted by statutory regulation or exceeds the permitted use, you will need to obtain permission directly from the copyright holder. To view a copy of this license, visit http://creativecommons.org/ licenses/by/4.0/.

(C) The Author(s) 2019 